\title{
Precipitation mechanisms of micro-crystal graphite powder during type IIa large diamonds crystallization process
}

\author{
Shangsheng Li ${ }^{\star}{ }^{a}$, Chunsheng Gonga, Taichao Sua Menhua Hua ${ }^{a}$, He Zhanga, \\ Hongan Mab, Xiaopeng Jiab
}

(a. School of Materials Science and Engineering, Henan Polytechnic University, Jiaozuo, Henan 454000, China;

b. State Key Laboratory of Superhard Materials, Jilin University, Changchun, Jilin; 130012, China;)

Corresponding author: Shangsheng Li

Email: lishsh@hpu.edu.cn

\section{Abstract}

Type Ila large diamond crystals were synthesized in FeNiCo-C system with $\mathrm{Ti}(\mathrm{Cu})$ as nitrogen getter by temperature gradient method (TGM) under high pressure and high temperature (HPHT) conditions. Diamonds grew upon (111) facet under $5.4 \mathrm{GPa}$ and $1620 \mathrm{~K}$ while black powder appeared around the growing diamond crystal. And the black powder covered the growing crystals in cap shape and restrained the diamond growth drastically with growth time prolonged. X-ray diffraction (XRD) and scanning electron microscopy (SEM) were utilized to characterize the black powder described above and the regrown graphite obtained from the growth process of traditional type Ib diamonds. The black powder was proved to be micro-crystal graphite powder and the powder was similar to the regrown graphite. However, there were obvious differences between the micro-crystal graphite powder and the regrown graphite. The present discussion about precipitation mechanisms of micro-crystal graphite powder implied that the added $\mathrm{Ti}(\mathrm{Cu})$ and related reaction-products in FeNiCo-C system could change solvent property of the catalyst, which affected solubility curve of graphite. Furthermore, the practice of diamond growth indicated that the relatively high temperature and relatively low pressure 
conditions were remarkably responsible for the precipitation of micro-crystal graphite powder.

Keywords: diamond crystal; HPHT; micro-crystal graphite

\section{Introduction}

Natural diamonds contain many defects and stress concentrations derived from severe growth environment. An overwhelming majority of natural diamonds corresponds to type Ia with high nitrogen content. It is quite difficult to find high-quality diamond crystals free from defects or stresses, even among rare natural type IIa diamonds. The controllable diamond growth under HPHT conditions makes it attainable to obtain high-quality type IIa diamonds ${ }^{1-3}$. High-purity, perfect-crystallization type Ila large diamond crystals (diameter $>1$ $\mathrm{mm}$ ) without defects can be applied in many fields ${ }^{1-10}$.Therefore, it is meaningful to research the synthesis of type IIa large diamond crystals.

It is well known that large diamond crystals are generally synthesized by temperature gradient method (TGM) developed by GE Company in 19711-3. In this method, the carbon positioned at high temperature region became diamond and dissolved into the melting catalyst at HPHT conditions. Then the dissolved carbon was forced by a proper temperature gradient to diffuse to the low temperature region and finally crystallized as diamond upon the surface of seed crystal. Compared with traditional type Ib large diamond crystals, type IIa large diamond crystals were more difficult to grow, because nitrogen getter usually resulted in inclusions involved into diamond structures or produced craters on the surface of diamond crystal. Hence, limiting growth rate as an optimized synthesis process was necessary to avoid the above problems ${ }^{9-13}$.

Generally speaking, if the growth rate was too high or the synthesis condition was so-called "relatively high temperature and relatively low pressure", the excessive carbon tended to crystallize as graphite instead of diamond. The graphite has no enough time to form diamond during growth process of traditional type Ib large diamond crystals ${ }^{14-15}$. Zang et al. studied the formation mechanisms of regrown graphite formed during the growth process of 
traditional type Ib large diamond crystals and the effects of regrown graphite on crystal growth of type Ib diamond ${ }^{15}$. In the present study, black powders, similar to regrown graphite, were generated and restrained diamond growth drastically if type IIa large diamond crystals grew slowly at a relatively high temperature. However, there was a lack of the study about the black powders. In this paper, precipitation mechanisms of the black powders obtained from the crystallization process of type IIa large diamond crystals by TGM were discussed in details. Additionally, a route for eliminating the black powders was proposed to synthesize high-quality type IIa large diamond crystals.

\section{Experiment}

Experiments of diamond growth were carried out in a China-type cubic high-pressure apparatus (SPD6×1200MN), using high-purity graphite as carbon source, FeNiCo alloy as catalyst, high-quality cub-octahedral diamond single crystal with a diameter of $0.6 \mathrm{~mm}$ as seed crystal, (111) facet as growth surface. High-purity Ti foil was added into the catalyst to trap nitrogen, and meanwhile $\mathrm{Cu}$ was added to decompose TiC.

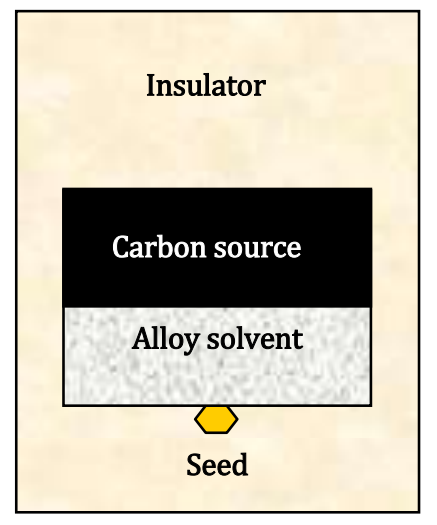

Fig. 1 The schematic diagram of the growth chamber

The schematic diagram of reaction cell with a diameter of $10 \mathrm{~mm}$ in graphite heater was shown in Fig.1. Based on the practical demand, the growth rate of diamond crystals could be changed by adjusting the axial temperature gradient in reaction cell1-5. Pressures were calculated by the relationship between the oil load and actual pressure in the compressed reaction cell, which was calibrated 
by investigating the electric resistance sudden changing resulted from the phase transitions in some reference materials, such as $\mathrm{Bi}, \mathrm{Ba}$ and $\mathrm{Tl}^{1-3}$. Temperatures were calculated by the relationship between the power input and actual temperature in the compressed reaction cell, which was measured by $\mathrm{Pt} 6 \% \mathrm{Rh} /$ Pt30\% Rh thermalcouple ${ }^{1-3}$.

\section{Results and discussion}

\subsection{The precipitation of black powder}

The crystallization experiments of type Ila large diamond crystals (111)-orientated were run in FeNiCo-C system with $1.5 \mathrm{wt} \% \mathrm{Ti}(\mathrm{Cu}) \quad(\mathrm{Ti}$ and $\mathrm{Cu}$ respectively accounted for $1.5 \%$ of the catalyst weight) at pressure of $5.4 \mathrm{GPa}$ and temperature of $1620 \mathrm{~K}$. The growth rate must be strictly controlled about $3 \mathrm{mg} / \mathrm{h}$ to avoid the inclusions incorporation into diamond structure and the craters generation on diamond surfaces due to the addition of nitrogen getter ${ }^{1-3}$. Therefore, in order to synthesize high-quality type Ila large diamond crystals, the axial temperature gradient in the reaction cell was modified so that the growth rate of type IIa diamond was much lower than that of type Ib diamond. The parameters of large diamond crystal growth are listed in table 1. It was found that some black powders (Seen in Fig 2) was collected from the sample after acid treatment for a short-time (10h) and the amount of black powders increased gradually with growth time. A large amount of black powders surrounded the diamond crystal in the shape similar to cap (Seen in Fig 3) and restrained its growth drastically if the growth time arrived 30h. However, no black powder was got after a similar synthesis $(30 \mathrm{~h})$ without $\mathrm{Ti}(\mathrm{Cu})$ additives at the same conditions. As shown in Fig. 4, it was noticed that a large amount of regrown graphite was collected after the similar synthesis (30h) under pressure of 5.4 GPa and at temperature of $1620 \mathrm{~K}$ (discussed in section 3.2). The production of regrown graphite was caused by enhancing the temperature gradient. 
Table 1. The parameters of large diamond crystal growth in Fig.2 Fig.4

\begin{tabular}{|c|c|c|c|c|c|}
\hline & Growth Time & Crystal Weight & Graphite Weight & Axial temperature & Diamond Growth \\
\hline Sample & $\mathrm{h}$ & $\mathrm{mg}$ & $\mathrm{mg}$ & gradient $\mathrm{k} / \mathrm{mm}$ & rate $\mathrm{mg} / \mathrm{h}$ \\
\hline Fig.2 & 10 & 30 & 6 & 15 & 3.0 \\
\hline Fig.3 & 30 & 84 & 21 & 15 & 2.8 \\
\hline Fig.4 & 30 & 186 & 26 & 30 & 6.2 \\
\hline
\end{tabular}

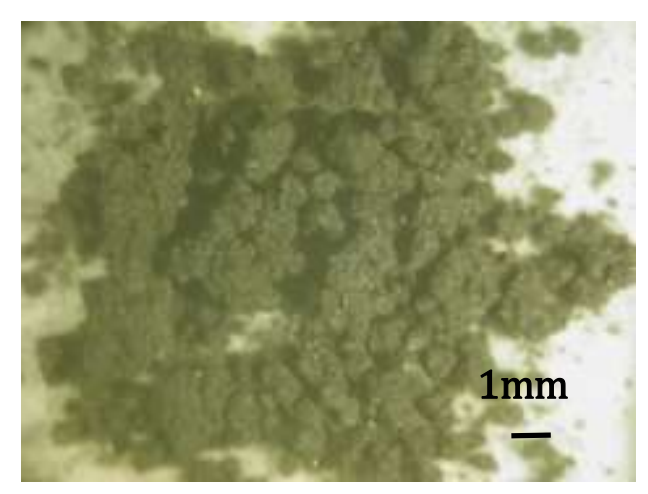

Fig.2 The black powders of type IIa diamond grown for $10 \mathrm{~h}$

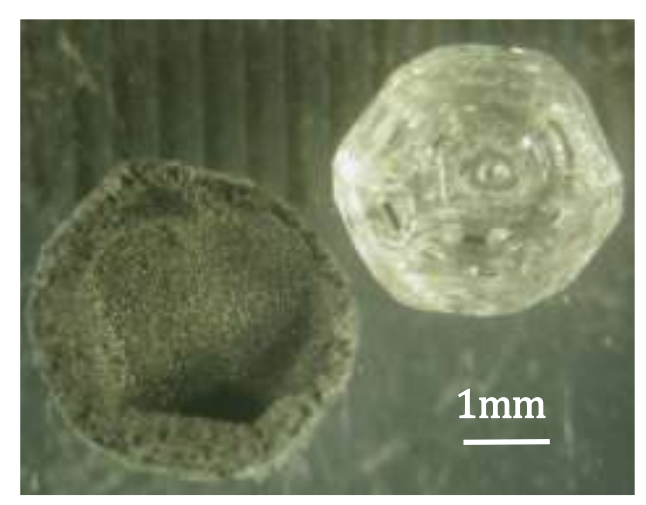

Fig.3 Type IIa diamond grown for $30 \mathrm{~h}$ and the black powders in the shape similar to cap 


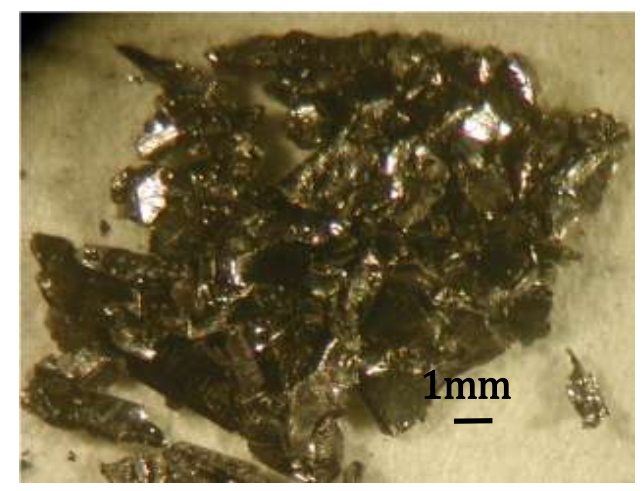

Fig.4 Regrown graphite formed during growth process for 30h of type $\mathrm{Ib}$ diamond

\subsection{Characterizations by XRD and SEM}

It is shown in Fig. 5 that black powders (Seen in Fig 3) collected after acid treatment against the catalyst used for the growth of type IIa diamond was proved to be ordinary graphite with a little $\mathrm{Ti}_{2} \mathrm{O}_{3}$. Figure 6 shows the XRD pattern of regrown graphite (Seen in Fig 4) generated in the growth process of type Ib diamond. Considering that black powders were collected after acid treatment, we used XRD to measure the HPHT-treated catalyst before acid treatment got from growth process of type Ila diamond (Fig. 7) and to investigate the complex reactions related with additives in the synthesis system. Fig. 7 indicates that $\mathrm{Cu}$, TiN and $\mathrm{Ti}_{2} \mathrm{O}_{3}$ remained in the FeNiCo catalyst after the HPHT process.

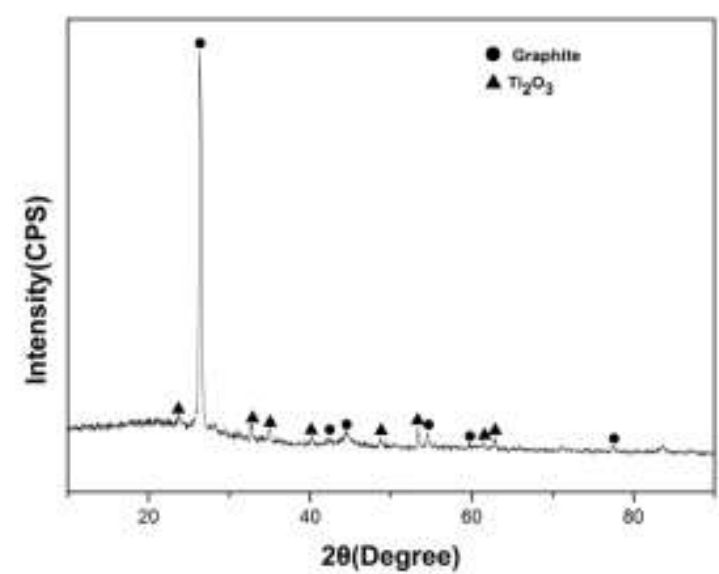

Fig.5 XRD pattern of black powders collected from growth process of type IIa diamond 


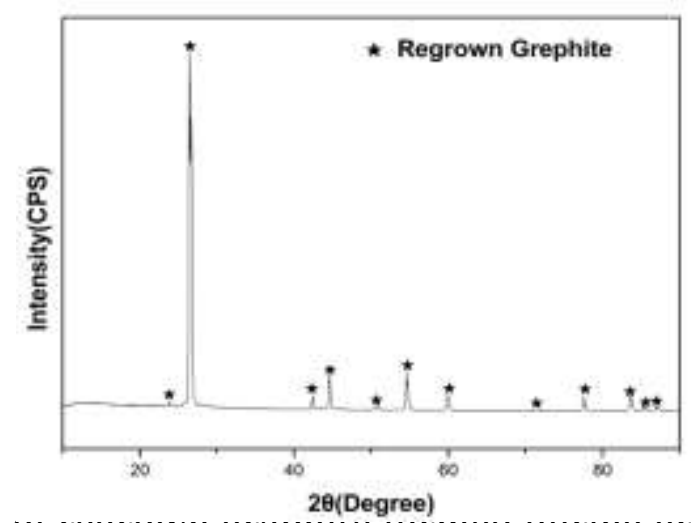

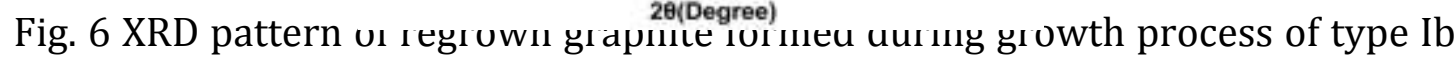
diamond

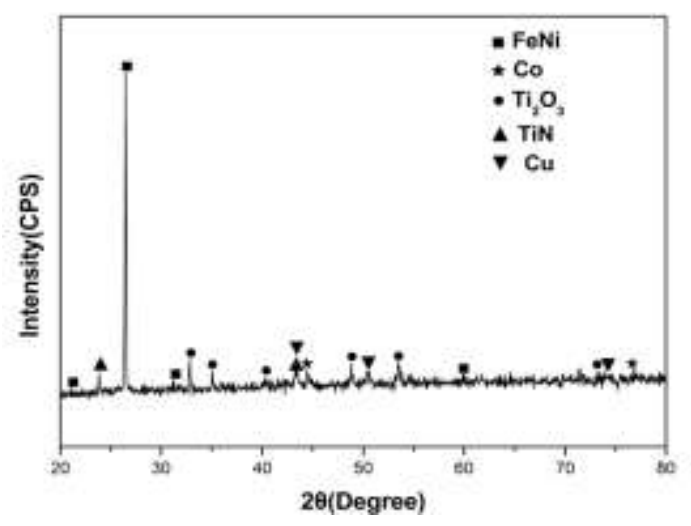

Fig.7 XRD pattern of the HPHT-treated catalyst got from growth process of type Ila diamond before acid treatment

As shown in Fig. 8 (a) and (b), black graphite powders (Seen in Fig 3) were in flake shape. The dimensions were several micrometers in diameter. Regrown graphite (Seen in Fig 4) exhibited in a large sheet shape with smooth surface, as shown in Fig. 8 (c) and (d).

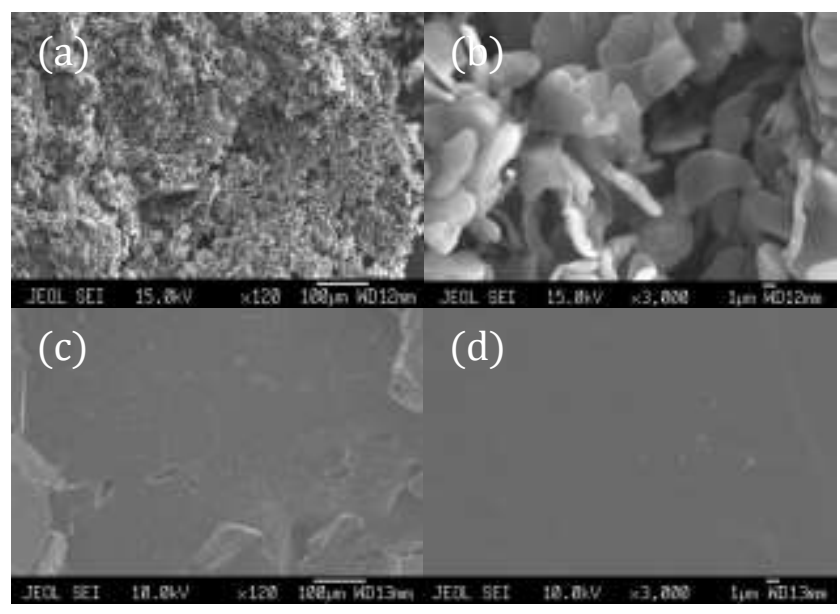

Fig.8 SEM images of (a), (b) black graphite powders and (c), (d) regrown graphite 
According to the results of XRD and SEM, black powders and regrown graphite were the same in phase structure but different in size and shape. The black powders precipitated from type IIa large diamond growth process was called the micro-crystal graphite in above test. Both micro-crystal graphite and the regrown graphite produced from the growth of type Ib large diamond were graphite phase. However, the difference between them was that the former graphite did not grow up continuously; the latter was the precipitation of large sheet graphite crystals.

\subsection{Precipitation mechanism of micro-crystal graphite powder}

Zang et al. discussed the precipitation mechanisms of regrown graphite formed during a fast growth process of type Ib diamond on the basis of solubility curves of graphite and diamond in the catalyst ${ }^{14,15}$. Their discussion is helpful for us to research the micro-crystal graphite.

In our study, the difference of micro-crystal graphite powder and regrown graphite is that the former occurred during a slow growth process at high temperature of type IIa large diamond crystals upon (111) facet. Basing on the results above, the micro-crystal graphite powder had been turned out to be distinct with regrown graphite. According to viewpoints from Zang et al., a slow growth process for type IIa large diamond crystals would not lead to formation of graphite, but additives in the synthesis system for trapping nitrogen may react (Seen in Fig 7) as follows 16:

$$
\begin{gathered}
\mathrm{Ti}+\mathrm{N} \rightarrow \mathrm{TiN} \\
\mathrm{Ti}+\mathrm{C} \rightarrow \mathrm{TiC} \\
\mathrm{TiC}+\mathrm{Cu} \rightarrow \mathrm{Ti}^{-}+\mathrm{Cu}^{+}+\mathrm{C}
\end{gathered}
$$

According to the XRD result shown in Fig.7, one more reaction may exist as follows:

$$
4 \mathrm{Ti}+3 \mathrm{O}_{2} \rightarrow 2 \mathrm{Ti}_{2} \mathrm{O}_{3}
$$

It was the existence of additives and related products that changed the property of catalyst-solvent. Especially for $\mathrm{Cu}$, its low viscosity and weak solvent power made the saturation of graphite decrease. These are the reasons that 
made the difference of solubility between diamond and graphite in the catalyst small. Thus the two curves approached each other (Fig. 9) ${ }^{15}$. As a result, if a slow growth process for type IIa diamond ran under a "relatively low pressure and relatively high temperature" condition, carbon source precipitated upon the surface of seed crystal as diamond, meanwhile metastable graphite also precipitated and remained around the growing crystal. Owing to the existence of additives $\mathrm{Ti}(\mathrm{Cu})$ and its related products, the growth of graphite is prevented so that micro crystal graphite powders appear.

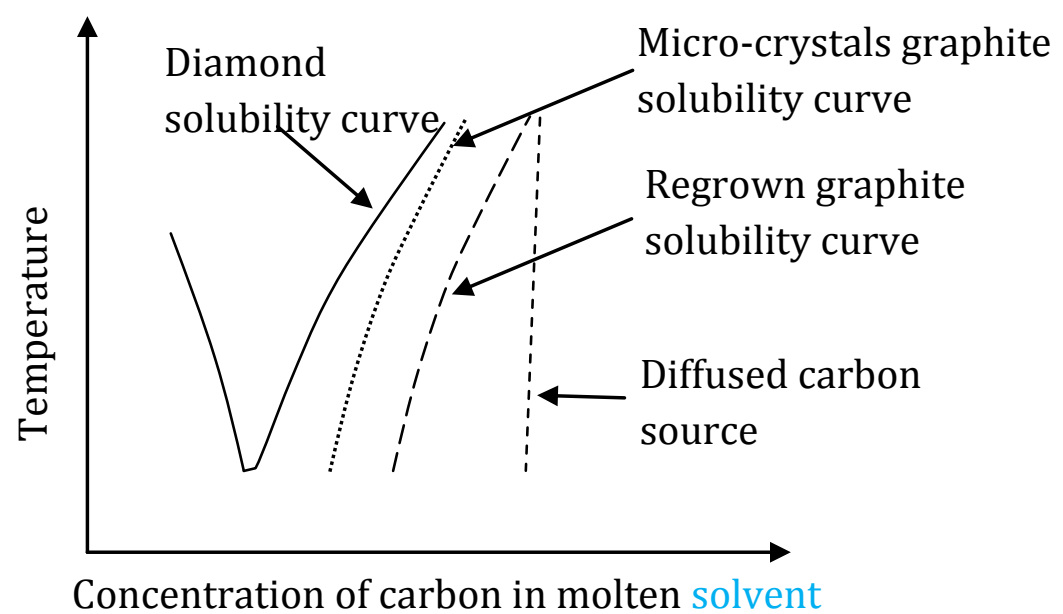

Fig.9 Solubility difference schematic diagram of micro-crystals graphite and regrown graphite in molten metal solvent

Close to the condition for type Ib diamond growth which regrown graphite came out, "relatively high temperature and relatively low pressure" was the necessary condition for the precipitation of as-described micro-crystal graphite powders. Since the additives were essential for growing type IIa large diamond crystals, decreasing the synthesis temperature and increasing the synthesis pressure must be adopted to eliminate micro-crystal graphite.

\subsection{Diamond synthesis after decreasing the temperature and increasing the} pressure 
As discussed in 3.3 , the additives for trapping nitrogen and the "relatively high temperature and relatively low pressure" of conditions are necessary for the precipitation of micro crystal graphite. Since nitrogen must be removed for growing type IIa large diamond crystals, the additives of nitrogen getter are essential. Therefore, it was effective for eliminating the micro-crystal graphite on diamond growth to decreasing the temperature and increasing the pressure. With no change on other parameters of the synthesis conditions, the temperature and pressure are adjusted as $1510 \mathrm{~K}$ and $5.8 \mathrm{GPa}$ for growing type IIa large diamond crystals. Consequently, a high-quality type IIa large diamond crystal, $4.3 \mathrm{~mm}$ in diameter, has been synthesized after 30h (Fig.10). By taking the above measures, the micro-crystal graphite was not precipitated. Then the growth of diamond was faster than that of large diamond growth with micro crystal graphite precipitation as shown in Fig. 3.

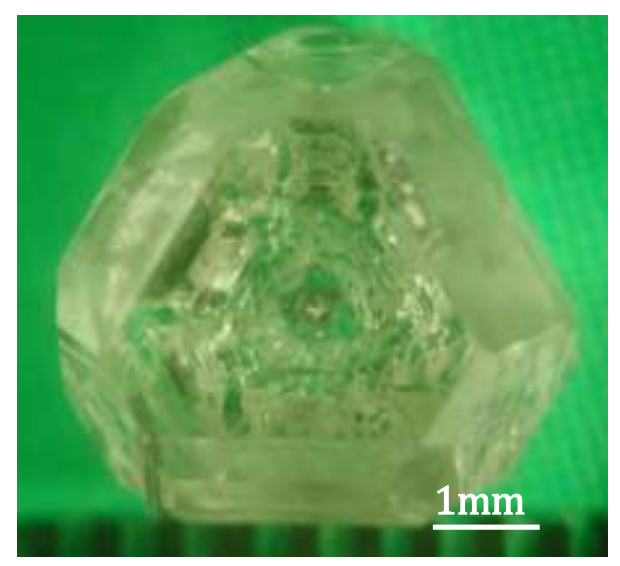

Fig.10 Type IIa diamond crystal synthesized for $30 \mathrm{~h}$ after decreasing the temperature and increasing the pressure

Table 2 summarizes the different growth conditions of different type diamonds and the XRD results, morphology/composition of the black powder precipitated with different type diamonds. These results implied that low pressure condition was inappropriate for growing type Ila high-quality diamond. Relatively, it required a higher pressure for type IIa large diamonds than the pressure for type Ib large diamond crystals to avoid the precipitation of micro crystal graphite. This viewpoint was consistent with the idea previously reported 
by Sumitomo ${ }^{3}$. Compared with type Ib large diamond crystals, type IIa large diamond crystals correspond to a new synthesis region with the lifted lower boundary ${ }^{1-3}$.

Table 2. Experiment and measurement results for type IIa and Ib large diamonds

\begin{tabular}{|c|c|c|c|c|c|}
\hline Sample type & $\begin{array}{l}\text { Diamond Growth } \\
\text { rate }(\mathrm{mg} / \mathrm{h})\end{array}$ & $\begin{array}{l}\text { Pressure } \\
\text { (GPa) }\end{array}$ & Temperature & $\begin{array}{c}\text { Black powder } \\
\text { Morphology/SEM }\end{array}$ & $\begin{array}{l}\text { Black powder } \\
\text { Composition/XRD }\end{array}$ \\
\hline Type IIa (Fig.3) & 2.8 & 5.4 & 1620 & Small flake & $\begin{array}{l}\text { ordinary graphite } \\
\text { with a little } \mathrm{Ti}_{2} \mathrm{O}_{3}\end{array}$ \\
\hline Type Ib (Fig.4) & 6.2 & 5.4 & 1620 & Large sheet & regrown graphite \\
\hline Type IIa (Fig.10) & 2.8 & 5.8 & 1510 & no & no \\
\hline
\end{tabular}

\section{Conclusions}

Based on the characterization of black powders formed during the growth process of type IIa large diamond crystals, conclusions were summarized as follows:

(1) The black powders were micro-crystal graphite powder and had undesirable effects on the growth of type Ila large diamond crystals.

(2) Additives for trapping nitrogen and related products could change the property of catalyst-solvent, and result in similar solubility of diamond and graphite. Therefore metastable micro-crystal graphite precipitates and remains.

(3) The "relatively high temperature and relatively low pressure" condition was also a noticeable reason for the precipitation of micro-crystal graphite

\section{Acknowledgments}

This work is sponsored by National Science Foundation of China (Grant No.51172089); Education Department of Henan Province, China (Grant No.12A430010); Program for Innovative Research Team of Henan Polytechnic University (Grant No.T2013-4); Fundamental Research Funds for the Universities of Henan Province(Grant No.NSFRF140110); Doctoral Fund of Henan Polytechnic University, China (Grant No.B2013-013). 


\section{References:}

1 H. M. Strong, R. M. Chrenko, J. Phys. Chem. 1971, 75, 1838.

2 H. Sumiya, S. Satoh, Diamond Rel. Mater, 1996, 5, 1359.

3 H. Sumiya, N. Toda, S. Satoh, J Cryst Growth, 2002, 237-239, 1281.

4 Q. G. Han, B. Liu, M. H. Hu, Z. C. Li, X. P. Jia, et.al, Cryst Growth Des. 2011, 11, 1000-1005.

5 S. S. Li, H. A. Ma, X. L. Li, T. C. Su, G. F. Huang, Y. Li, et.al, Chin. Phys. B. 2011, $20,028103$.

6 Y. Li, X. P. Jia, H. A. Ma, J. Zhang, F. B. Biao, et.al, CrysEngComm. 2014, 16, 7547-7551

7 M. H. Hu, N. Bi, S. S. Li, T. C. Su, X. P. Jia, H. A. Ma, et.al, Chin. Phys. B.2015,24, 038101.

8 S. S. Sun, X. P. Jia, B. M. Yan, F. B. Biao, N. Chen, et.al, CrysEngComm. 2014, $16,2290-2297$.

9 H. Sumiya, N.Toda, Y. Nishibayashi, S. Satoh, J Cryst Growth. 1997, 178, 485-494.

10 Z. F. Zhang, X. P. Jia, S. S. Sun, X. B. Liu, Y. Li, B. M. Yan, et.al, Int J of Refract Met Hard Mater. 2013, 38, 111-117.

11 G. F. Huang, X. P. Jia, Y. J. Wen, H. A. Ma, Z. Y. Jin, Int J of Refract Met Hard Mater. 2013, 41, 517-521.

12 C.M. Li, R.H. Zhu, J.L. Liu a, L.X. Chen, J.C. Guo, C.Y. Hua, et.al, Diamond Rel. Mater. 2013, 39, 47-52.

13 R. C. Burns, J. O. Hansen, R. A. Spits, M. Sibanda, C. M. Welbourn, D. L. Welch, Diamond Rel. Mater. 1999, 8, 1433-1437.

14 Hanneman, J. Chem. Phys. 1967, 9, 3668.

15 C. Y. Zang, X. P. Jia, H. A. Ma, Y. Tian, H. Y. Xiao, Chin. Phys. Lett, 2005, 9, 2415. 
16 S. S. Li, X. P. Jia, C. Y. Zang, Y. TIAN, Y. F. Zhang, H. Y. Xi, et.al, Chin. Phys. Lett, 2008, 10, 3801-3804. 